\title{
Venus scientists fear neglect
}

Researchers say that infrequent visits are hindering studies of the nearest planet.

\section{BY ERIC HAND}

$\mathrm{V}$ enus would seem to be a tempting destination for planetary probes: conveniently close, and an extreme laboratory for atmospheric processes familiar on Earth. So why won't NASA send a mission there? That was the frustrated question coming from scientists at the annual meeting of NASA's Venus Exploration Analysis Group (VEXAG) near Washington DC on 30-31 August. They perceive an agency bias against Venus, a planet that hasn't seen a US mission since the Magellan probe radar-mapped its shrouded surface in the early 1990s, and which won't see one any time soon, after NASA this year rejected a bumper crop of Venus proposals.

"A lot of us are dismayed," says David Grinspoon, astrobiology curator at the Denver Museum of Nature and Science in Colorado, who is a co-investigator on several of the proposals. Some of the reasons for the planet's neglect are obvious: surface temperatures that would melt lead and thick clouds of sulphuric acid make data gathering a challenge for landers and orbiters alike. And - unlike Mars - Venus is neither a plausible haven for life nor a potential destination for astronauts.

But Grinspoon says that something more insidious is at work. Without new missions supplying data for analysis, funding for Venus research has dwindled, leading to fewer students entering the field - and a smaller constituency to lobby for missions. "Because of this feedback loop, the community has shrunk," he says. Research grants mentioning Venus have accounted for just $2 \%$ of NASA's planetary-science funding since 2005.

Internationally, things aren't much better. Europe's Venus Express, a probe cobbled together using instruments designed for missions to Mars and a comet, has only partly satisfied a craving for data since it arrived in 2006. And last December, Japan's Akatsuki spacecraft failed to enter orbit and overshot the planet.

In May, Venus researchers got a double dose of further bad news. In NASA's New Frontiers medium-class mission line, a mission to return asteroid samples prevailed over a proposed Venus lander that would have lasted a precious three hours on the surface. And there were no Venus missions among the three

\section{DNATURE.COM}

For more on Venus science, visit: go.nature.com/wjsaiw finalists in the Discovery low-cost planetarymission competition, although one-quarter of the proposals had

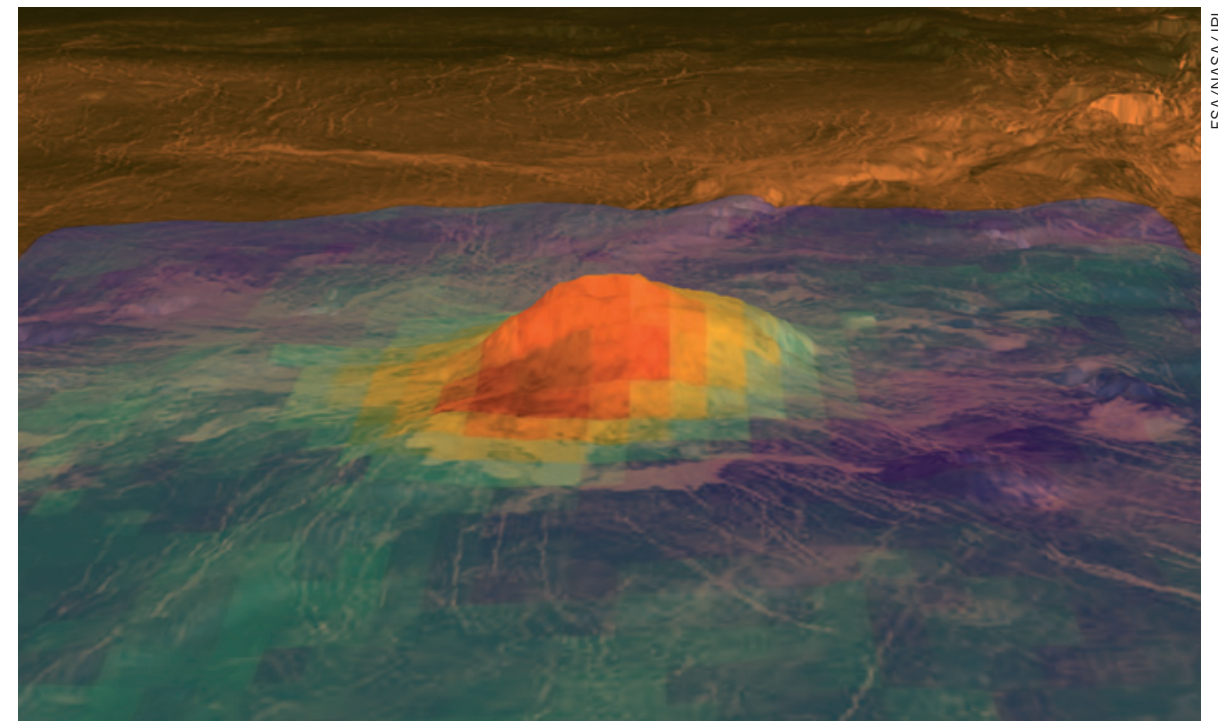

The volcano Idunn Mons shows up as a hot spot in this thermal map taken by the Venus Express probe.

targeted the planet (see 'Forgotten planet').

Of the seven Discovery proposals for Venus missions, reviewers gave six the lowest possible ranking, guaranteeing their rejection. Only one, an atmospheric mission, received a solid 'category II' score. With so many proposals, and with mission teams averaging 20 people each, some Venus scientists wondered whether enough unbiased colleagues were left in the community to competently review the proposals. But Jim Green, director of planetary science at NASA, says that he found plenty of qualified reviewers from outside the United States. "There were just better proposals" for

\section{FORGOTTEN PLANET}

Venus was targeted by one-quarter of the 28 proposals in NASA's latest Discovery competition. But no Venus missions were among the finalists.

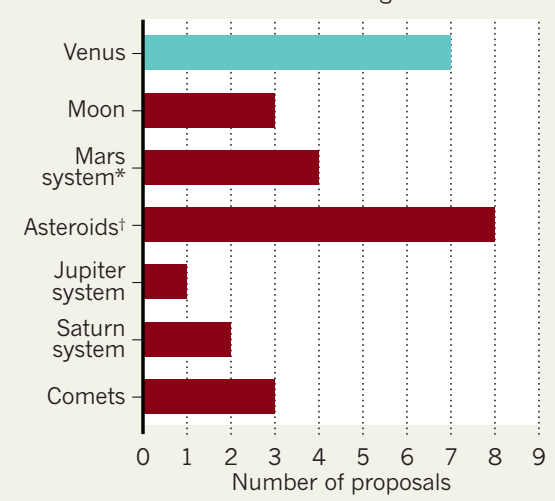

*Includes mission to visit the Mars moon Phobos. tIncludes mission to one of Jupiter's Trojan asteroids. other Solar System targets, he says.

Michael New, the NASA programme scientist who ran the competition, says that Venus scientists need a clearer consensus on their goals and the measurements that they want to make. Those who want to map the surface, for instance, have not determined how much better than Magellan their radar instruments have to be. NASA may invite another round of Discovery proposals in 2012.

Grinspoon hopes that by then, the unanswered scientific questions will be impossible to ignore. He wants to know why Earth's global climate models break down on Venus, which has an atmosphere composed of $97 \%$ carbon dioxide - and what that reveals about the hidden fine-tunings of Earth models.

Similarly, Gordon Chin, a project scientist at NASA's Goddard Space Flight Center in Greenbelt, Maryland, proposed a mission that would explain why the same chemical processes that destroy ozone in Earth's atmosphere stabilize carbon dioxide in Venus's. And Suzanne Smrekar, VEXAG co-chair and a scientist at NASA's Jet Propulsion Laboratory in Pasadena, California, wants to follow up on a 2010 finding in which she and her colleagues used Venus Express data to identify hot spots on the planet's surface - evidence for volcanism within the past few million years. A clearer picture of volcanism on Venus and its history could help to explain how the planet's runaway greenhouse effect got going. "We need another laboratory to test what we think we know on Earth," says Chin. - 